\title{
BMJ Open Study protocol for a randomised, double-blinded, placebo-controlled, clinical trial of $S$-ketamine for pain treatment in patients with chronic pancreatitis (RESET trial)
}

Jacob Juel, ${ }^{1}$ Søren Schou Olesen, ${ }^{1}$ Anne Estrup Olesen, ${ }^{1,2}$ Jakob Lykke Poulsen, ${ }^{1}$ Albert Dahan, ${ }^{3}$ Oliver Wilder-Smith, ${ }^{4,5}$ Adnan Madzak, ${ }^{6}$ Jens Brøndum Frøkjær, ${ }^{6,7}$ Asbjørn Mohr Drewes ${ }^{1,7}$

To cite: Juel J, Olesen SS Olesen AE, et al. Study protocol for a randomised, double-blinded, placebocontrolled, clinical trial of $S$-ketamine for pain treatment in patients with chronic pancreatitis (RESET trial). BMJ Open 2015;5:e007087. doi:10.1136/bmjopen-2014007087

- Prepublication history for this paper is available online. To view these files please visit the journal online (http://dx.doi.org/10.1136/ bmjopen-2014-007087).

Received 2 November 2014 Revised 16 February 2015 Accepted 17 February 2015

\section{CrossMark}

For numbered affiliations see end of article.

\section{Correspondence to}

Professor Asbjørn Mohr Drewes;

amd@mech-sense.com

\section{ABSTRACT}

Introduction: Chronic pancreatitis (CP) is an inflammatory disease that causes irreversible damage to pancreatic tissue. Pain is its most prominent symptom. In the absence of pathology suitable for endoscopic or surgical interventions, pain treatment usually includes opioids. However, opioids often have limited efficacy. Moreover, side effects are common and bothersome. Hence, novel approaches to control pain associated with $\mathrm{CP}$ are highly desirable.

Sensitisation of the central nervous system is reported to play a key role in pain generation and chronification. Fundamental to the process of central sensitisation is abnormal activation of the $\mathrm{N}$-methyl-D-aspartate receptor, which can be antagonised by $S$-ketamine. The RESET trial is investigating the analgaesic and antihyperalgesic effect of $S$-ketamine in patients with $\mathrm{CP}$.

Methods and analysis: 40 patients with $\mathrm{CP}$ will be enrolled. Patients are randomised to receive $8 \mathrm{~h}$ of intravenous $S$-ketamine followed by oral $S$-ketamine, or matching placebo, for 4 weeks. To improve blinding, $1 \mathrm{mg}$ of midazolam will be added to active and placebo treatment. The primary end point is clinical pain relief as assessed by a daily pain diary. Secondary end points include changes in patient-reported outcome measures, opioid consumption and rates of side effects. The end points are registered through the 4week medication period and for an additional follow-up period of 8 weeks to investigate long-term effects. In addition, experimental pain measures also serves as secondary end points, and neurophysiological imaging parameters are collected. Furthermore, experimental baseline recordings are compared to recordings from a group of healthy controls to evaluate general aspects of pain processing in $\mathrm{CP}$.

Ethics and dissemination: The protocol is approved by the North Denmark Region Committee on Health Research Ethics (N-20130040) and the Danish Health and Medicines Authorities (EudraCT number: 2013003357-17). The results will be disseminated in peerreviewed journals and at scientific conferences.

\section{Strengths and limitations of this study}

- The clinical efficacy, and the experimental investigation of the underlying mechanisms of the antihyperalgesic and analgaesic properties of $S$-ketamine, are both addressed in this trial.

- This is a single-centre trial, which may compromise the external validity of the findings. However, single-centre trials also have several advantages. They are often logistically easier and data collection is simpler, and they typically deal with a less heterogeneous population, thereby diminishing confounding.

Trial registration number: The study is registered at http://www.clinicaltrialsregister.eu (EudraCT number 2013-003357-17).

\section{INTRODUCTION}

Chronic pancreatitis (CP) remains a major source of morbidity in Northern Europe, with an annual incidence of approximately 10 per 100000 inhabitants. ${ }^{1}$ A typical cause is long-term excessive use of alcohol, although genetic, environmental and autoimmune factors have also been associated with CP. It is a disease characterised by progressive destruction of the pancreatic gland and, as the disease evolves, significant impairment of exocrine as well as endocrine functions takes place. Within 5 years of diagnosis, endocrine and exocrine insufficiencies develop in approximately $50 \%$ and $80 \%$ of patients with CP, respectively. These conditions are usually managed sufficiently with antidiabetic treatment and pancreatic 
enzymes to optimise metabolic and nutritional status, whereas the treatment of pain in CP is more intricate. This progressive destruction also leads to pain, which is the most common symptom in CP, and up to $90 \%$ of patients have chronic abdominal pain, often worsened by acute pain exacerbations typically requiring hospitalisation. ${ }^{2}$ Hence, pain is a major burden for most patients with $\mathrm{CP}$ and it has been associated with impaired psychosocial functioning, physical disability and decreased life quality. ${ }^{3}{ }^{4}$ It is recognised that chronic pain may alter central pain processing, for example, central sensitisation, as the continuous damage of the pancreatic nerves may in time lead to central sensitisation of the pain system. The key component in this process is aberrant activation of the $N$-methyl-D-aspartate (NMDA) receptor. The initial analgaesic medication in painful CP will often involve opioids in the absence of pathology suitable for endoscopic or surgical interventions. However, opioid-based analgaesia often only shows limited effectiveness in these patients and it is frequently accompanied by undesirable side effects. ${ }^{5}$ However, an NMDA receptor antagonist, for example, S-ketamine, could potentially be able to reverse this central sensitisation by its action on the NMDA receptor, thus providing long-term pain relief in sufferers of chronic pain. ${ }^{6}$

\section{Pain mechanisms in CP}

The pathophysiology of pain in CP has yet to be fully elucidated; it is probably of multifactorial origin. Historically, pain treatment has focused on the pancreatic gland, assuming pain to be generated by ongoing pancreatic inflammation, parenchymal hypertension and ductal obstruction. Consequently, treatment was focused on pathology in or closely related to the pancreatic gland. However, there is no direct relationship between abdominal pain and pancreatic morphology, and the experimental evidence supporting this is conflicting. ${ }^{7}$ The most recent explanation model of pain pathogenesis in $\mathrm{CP}$ is that recurrent inflammation beyond a certain threshold causes irreversible injury to the pancreatic tissue. ${ }^{8}$ This process of repeated inflammation is linked to continuous damage of the pancreatic nerves along with peripheral and central sensitisation of the pain system. Key to the process of central sensitisation is aberrant activation of the NMDA receptor as described below. An important outcome of central sensitisation is that once the disease has advanced and the neural pathophysiological processes are firmly established, the generation of pain becomes self-perpetuating and independent of the initial nociceptive drive. Consequently, the management of pain becomes difficult and conventional treatment much less effective. This novel and improved understanding of the pain aetiology advocates a paradigm shift in pain management of CP. ${ }^{610}$

\section{S-ketamine and central sensitisation}

Developed in the 1960s as an anaesthetic drug, S-ketamine is currently used not only as a safe anaesthetic drug, but also as an analgaesic drug in acute and preoperative pain as well as an antihyperalgesic drug in various chronic pain conditions. ${ }^{11}$ The different effects are dose dependent. It is classified as a non-competitive NMDA receptor antagonist, but acts on multiple receptors including opioid receptors. ${ }^{12}$ Hence, analgaesia induced by $S$-ketamine may be partly mediated by actions on opioid receptors; however, the main analgaesia is induced by the NMDA receptor antagonism. Ketamine exists in two distinct stereoisomeric forms, the S-form and the R-form. A racemic equimolar mixture and a pure S-enantiomer are available. The S-isomeric form has a four times higher affinity for the NMDA receptor compared to the R-isomeric form and will be used in the present study. ${ }^{12}$ In anaesthesia, S-ketamine provides a dissociative anaesthetic state by blocking the connections between the limbic system and thalamus, while it provides an analgaesic and antihyperalgesic effect when used in subanaesthetic doses. The latter is mainly attributed to antagonism of the NMDA receptor, an excitatory inotropic glutamate receptor located in the central as well as the peripheral nervous system. At resting membrane potentials, the NMDA receptor ion channel is physically blocked by a magnesium ion so that no current flows if glutamate binds to it. Hence, activation of the NMDA receptor by glutamate produces excitation only when this magnesium block is relieved by depolarisation. Prolonged NMDA receptor activation results in removal of the magnesium ion blockade and a progressive increase in neuronal output. With ongoing activity, such activity-dependent sensitisation of central pain pathways ultimately becomes independent of the peripheral nociceptive drive and self-perpetuating. The mechanisms underlying this activity-independent sensitisation involves phosphorylation of different second messenger systems along with alterations in gene transcription. The resulting chronification of pain typically manifests as hyperalgesia and allodynia, which are the clinical hallmark of central sensitisation although not specific for this entity. Central sensitisation of the pain system is well documented in CP and it is increasingly accepted to play a prominent role in its pain pathogenesis. ${ }^{13-15}$

S-ketamine exerts its antihyperalgesic effect by restoring the NMDA receptor to its resting state condition through non-competitive antagonism. Thereby the 'gain' of the pain system is restored to normal physiological status. Multiple studies have consistently produced positive results regarding the use of S-ketamine in patients with chronic pain. Thus it comprises an interesting remedy to depress central sensitisation and its associated hyperalgesia in painful CP. This was supported by a recent Dutch double-blinded crossover trial designed to evaluate the effect of $S$-ketamine infusion on hyperalgesia associated with CP. Infusion of S-ketamine temporarily reversed pressure pain hyperalgesia and the underlying sensitised state of the pain system. This study was, however, not powered or designed for clinical end points. $^{16}$ 


\section{Experimental testing}

The experimental testing of the study is based on quantitative sensory testing and provides information of the pain on central as well as peripheral levels of the nervous system using controlled external pain stimuli. This evaluation of the sequential activation of the pain systems at different levels provides valuable information regarding the neuroplasticity in a sensitised nervous system. Assessment of experimental pain measures will be employed at baseline, during S-ketamine infusion, after 4 weeks of treatment with oral S-ketamine and at follow-up 8 weeks after treatment, to unravel the mechanisms of the underlying antihyperalgesic and analgaesic effects of Sketamine. Furthermore, experimental baseline assessments will be compared to assessments from a group of healthy controls to evaluate general aspects of pain processing in CP. The entities are summarised in box 1.

\section{Hypothesis}

We hypothesise that a 1 day infusion of $S$-ketamine followed by oral $S$-ketamine for 4 weeks decreases central

\section{Box 1 Primary clinical end points}

- The primary efficacy parameter is pain relief. This efficacy is assessed as changes in the daily experience of pain, which will be measured using a patient pain diary based on the visual analogue scale (VAS). Maximum pain intensity and average daily pain will be recorded at set times every day during the study.

Secondary clinical end points

- The ratio of responders versus non-responders defined by a decrease in VAS $>30 \%$ after 4 weeks compared to baseline.

- Change in opioid consumption.

- Change in quality of life using the European Organization for Research and Treatment of Cancer Quality of Life Questionnaire (EORTC-QLQ-C30).

- Changes in pain and physical functioning composite scores of the modified brief pain inventory-short form.

- Change in Izbicki pain score.

- Patient Global Impression of Change (PGIC).

- Change in Beck's Depression Inventory (BDI) to track changes in depressive symptoms.

- Change in Edmonton Symptom Assessment System (ESAS) for monitoring side effects and tolerability.

Experimental pain testing

- Pressure stimulation of the pancreatic viscerotome, control areas and the quadriceps.

- Tetanic electric stimulation of the pancreatic viscerotome and control area.

- Temporal summation to repetitive electric stimulations on the pancreatic and a control area.

- Conditioned pain modulation.

- Resting state EEG.

- Contact heat evoked potentials (EPs) on the pancreatic viscerotome and a control area.

- Somatosensory EPs with recovery cycle estimation on the median nerve.

- Nociceptive reflexes.

Offset-analgaesia with recording of EPs. sensitisation associated with CP and thereby induces clinical pain relief, which is also reflected in secondary end points and sensory testing, as summarised in box 1 .

\section{METHODS AND ANALYSIS \\ Concomitant medication}

Patients will be instructed not to change their regular pain treatment during the trial period. Rescue pain medication, taken on an 'as needed basis', is allowed throughout the trial period and its use will be documented in the daily pain diary.

\section{Recruitment}

All eligible patients with CP from our outpatient clinic who agree to participate in the study and fill in an informed consent will be invited to participate in the study. Patients will be recruited via personal correspondence and during sessions in the outpatient department, thus the initial contact will be in these settings.

\section{Randomisation}

Participants will be randomised to the study provided, they fulfil the entry criteria at screening (see boxes 2 and 3). A computer-generated pseudorandom code will be used to assign participants to treatment arms. A block randomisation will be used, allowing eight participants at a time to be randomised in equal proportions for S-ketamine or matching placebo.

\section{Study overview}

Participation in the study will involve four clinical visits as shown in figure 1 . The baseline visit, 1 week prior to the first administration of study medication, includes written informed consent, a physical examination and experimental pain testing. Furthermore, patients will be instructed in the use of a pain diary to record pain intensity on a daily basis. These assessments was given 7 days prior to medicine administration. Average and maximum daily pain intensities are assessed using the pain diary.

\section{Box 2 Inclusion criteria}

Patients above the age of 18 years with a diagnosis of chronic pancreatitis (CP) using the Mayo Clinic diagnostic criteria. Both the patients with and without diabetes will be allowed to enter the study.

- The participants must be able to read and understand Danish.

- The patients must suffer from chronic abdominal pain characteristic for $\mathrm{CP}$, meet the criteria for chronic pain (pain $\geq 3$ days/week for at least 3 months) and must consider their pain as insufficiently treated with their usual analgaesic treatment.

- Personally signed and dated informed consent document indicating that the patient has been informed of all pertinent aspects of the trial.

- Patients willing and able to comply with the scheduled visits, treatment plan, laboratory tests and other trial procedures. 


\section{Box 3 Exclusion criteria}

- Patients with any clinically significant laboratory abnormalities that in the opinion of the investigator may increase the risk associated with trial participation or may interfere with the interpretation of the trial results.

- Undiagnosed or untreated severe hypertension.

- Unstable angina.

Congestive heart failure.

- Any condition with elevated intracranial pressure.

- Untreated thyrotoxicosis.

- Alcohol dependence (alcohol use in accordance with the recommendations by the Danish Health and Medicines Authority is allowed).

- Illegal drug dependencies.

- Patients with evidence or history of medical or surgical disease of importance for this study as judged by the investigator.

- Patients treated with $S$-ketamine during the previous 4 months.

- Treatment with an investigational drug within 4 months preceding the first dose of study medication of importance for this study as judged by the investigator.

- Female patients who are pregnant or lactating, or intend to become pregnant, and male patients who intend to father a child during the course of the study. A pregnancy test will be conducted at baseline and after 4 weeks to ensure that female patients are not pregnant during the study medication period. The investigator will have to ensure that fertile female patients use a safe contraception method during the study and for at least $15 \mathrm{~h}$ after termination of the study medication period. The following methods are considered as safe contraception methods:

- The combined oral contraceptives

- Intrauterine device

- Gestagen injection

- Subdermal implantation

- Hormone vaginal ring

- Transdermal plaster

- Patients must not suffer from painful conditions other than CP that make them unable to distinguish the pain associated with $\mathrm{CP}$ from chronic pain of other origin.

- Patients with known hypersensitivity to $S$-ketamine or any of its components.

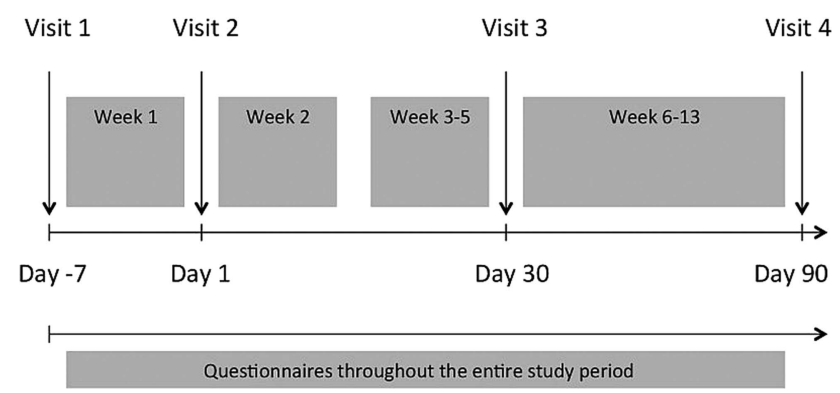

Figure 1 Visit 1 (baseline): Experimental testing and MRI.

Visit 2: Infusion and experimental testing. Visit 3:

Experimental testing and MRI. Visit 4 (end of study):

Experimental testing and MRI. Week 1: No study medication.

Week 2: Oral study medication. Ascending dosage of study medication. Weeks 3-5: Fixed dosage of study medication. Weeks 6-13: Follow-up for 8 weeks.
This is based on a visual analogue scale where 0 equals no pain and 10 equals the worst pain imaginable.

\section{Infusion of $S$-ketamine}

Duration and dosage of $S$-ketamine treatment were chosen based on a review of the literature and expert opinions as well as on feasibility considerations. At the second visit the patients will receive intravenous S-ketamine $(0.1 \mathrm{mg} / \mathrm{kg} / \mathrm{h})$ for $8 \mathrm{~h}$ or matching placebo (ie, isotonic saline). In addition, $1 \mathrm{mg}$ of midazolam will be administered together with ketamine/placebo to mask the patients' awareness of the central effects of ketamine and to ensure sufficient blinding.

\section{Preparation and administration of oral $S$-ketamine and placebo}

Oral solutions of S-ketamine and placebo will be prepared and provided by Skanderborg Apotek, Denmark. The placebo solution is similar to the vehicle used for ketamine solution. Thus, flavour and colour will match the characteristics of S-ketamine solution. During the second week of the study, patients will receive an increasing dose of oral $S$-ketamine or matching placebo starting on the day after S-ketamine infusion. The initial dose is $0.25 \mathrm{mg} / \mathrm{kg}$ S-ketamine three times per day. After 3 days, this will be increased to $0.50 \mathrm{mg} / \mathrm{kg} S$-ketamine three times per day, with a further increase to $0.75 \mathrm{mg} / \mathrm{kg}$ S-ketamine three times per day after 6 days and for the following 3 weeks. An equivalent dose-escalating regime will be followed in the placebo arm. All patients follow the same oral dosing schedule, with administration of study medication at 08:00, 14:00 and 20:00 $\pm 1 \mathrm{~h}$. If the patients experience unacceptable side effects, a single downward dose titration will be allowed, with the patient staying on that final dosage for the remaining study period. If side effects are intolerable on the minimum dose during the oral phase, participants have the option to drop out of the study, an option available to the participant at any stage of the study.

\section{Blood samples}

The following biochemical parameters will be monitored during the study:

- Alanine transaminase

- Aspartate transaminase

- Albumin

- Alkaline phosphatase

- Bilirubins

- $\gamma$-Glutamyl transpeptidase

- $\mathrm{C}$ reactive protein

- Urea

- Creatinine

- Haemoglobin

- Potassium

- International normalisation ratio

- Lactate dehydrogenase

- Sodium

- White cell count

- Thrombocytes 


\section{Study procedures}

Visit 1 (baseline)

At the baseline visit informed consent will be obtained for further progress in the study. After this is secured, physical examination will be conducted, blood samples drawn for biochemical screening and patients' medical history recorded. Moreover, a secretin-enhanced MR cholangiopancreatography will be performed to ensure that patients do not have any pathology suitable for endoscopic or surgical therapy. Next, instruction to all questionnaires will be given and reporting of daily pain intensity in the pain diary will begin. Quality of life will be registered using the European Organization for Research and Treatment of Cancer Quality of Life questionnaire (EORTC-QLQ-C30) ${ }^{17}$ while pain and physical functioning will be registered using the modified brief pain inventory-short form ${ }^{18}$ and Izbicki pain score ${ }^{19}$ and Patient Global Impression of Change (PGIC) is introduced for later use. ${ }^{20}$ Lastly Beck's Depression Inventory (BDI) is used to assess depressive symptoms ${ }^{21}$ and Edmonton Symptom Assessment System (ESAS) is used for monitoring side effects and tolerability. ${ }^{22}$ Daily, patients will report pain levels. Average and maximal pain will be assessed for a period of $24 \mathrm{~h}$ prior to recording. The use of rescue pain medication will also be reported in the diary. Furthermore, patients will have a thorough experimental pain examination at this visit (box 1). Finally, MRI of the brain will be carried out in relation to this visit to assess both functional and morphological entities.

\section{Visit 2}

At the second visit, the biochemical screening from visit 1 will be checked and questionnaires will be reviewed to secure compliance. Resting state EEG, contact heat evoked potentials on the pancreatic viscerotome (Th10) and a control area (Th4), and conditioned pain modulation will be performed before, during and after the infusion of S-ketamine or placebo. Fourteen blood samples will be drawn during the infusion in order to investigate the pharmacokinetics of $S$-ketamine and its metabolites.

\section{Monitoring between visits 2 and 3}

Patients are to be monitored closely during the entire study with frequent telephone interviews. Initially when medication is titrated, participants have 2-3 telephone interviews per week to monitor potential side effects and safety and to ensure compliance. During follow-up patients have at least one telephone interview allocated per week.

\section{Visit 3}

At the third visit, the experimental tests described for the first visit will be repeated. Pain diary and questionnaires will be collected. Blood for clinical chemistry will be drawn. Brain MRI of the cerebrum will be carried out as described in visit 1 . There are no further experimental medical interventions after this visit.

\section{Follow-up between visits 3 and 4}

During follow-up, the patients will be continually filling in their pain diary and questionnaires. The follow-up is employed to monitor the long-term effect of the study medication after the discontinuation of study medication.

\section{Visit 4 (end of study)}

The last visit takes place approximately 13 weeks after baseline. This visit is similar to the first and the third visit. All questionnaires will be filled in and collected. The experimental tests described for these visits will be repeated as well as brain MRI. After this visit patients are monitored as usual through our outpatient clinic.

\section{MRI studies}

The MRI studies of the cerebrum have been included in the protocol as an investigation at baseline, during ketamine or placebo treatment, and at the end of the study to assess any potential changes in the cerebrum.

\section{Subject withdrawal}

If the patient between the telephone calls experiences unacceptable adverse effects, the patient will be asked to contact the investigator. Based on a medical judgement, the dose of study drug may be reduced and the treatment continued or the patient may be withdrawn from the study. Follow-up consultations and eventually additional blood tests will be arranged as judged by the investigator.

\section{Discontinuation criteria}

If the participants are not able to tolerate the dose $0.25 \mathrm{mg} / \mathrm{kg}$ of $S$-ketamine three times per day during the oral phase of the study, they will be excluded. Otherwise, discontinuation will rely on the discretion of each participant. A healthy volunteer who discontinues will always be asked about the reason(s) for discontinuation and the presence of any adverse events. If needed, they will be seen and assessed by the investigator. New participants will replace dropouts.

\section{Dissemination}

Positive as well as negative and inconclusive results of the study will be reported in international peer-reviewed journals in the field of gastroenterology, pain or neurophysiology, and presented at conferences, hence dissemination to researchers as well as to clinicians will be achieved. Participants will be informed of the results of the trial by the investigators. Authorship will be ascribed in accordance with the Vancouver system. After the conclusion, a report will be submitted to the Danish Health and Medicines Authority and the North Denmark Region Committee on Health Research Ethics, as required by law.

\section{Safety considerations}

Some patients may experience nausea and dizziness for a brief period after administration. Moreover, adverse 
effects such as vivid dreams, nightmares and hallucinations have been described after anaesthesia and analgaesia with $S$-ketamine. ${ }^{23}$ Adverse effects are directly related to the dose used. In the present study, the doses are low and subanaesthetic, and severe adverse effects are unlikely to be seen. Adverse effects are self-assessed using a five-point Likert scale $(0=$ absent, $1=$ mild, $2=$ moderate, $3=$ severe, $4=$ very severe/unbearable) and the ESAS questionnaire. In regard to potential abuse of S-ketamine, we find the potential benefits in the treatment of chronic pain to outweigh the risk of recreational use of the drug. Almost every eligible participant for the trial are using opioid-based for analgesic purposes. This group of drugs have a large potential for abuse; however, there are no reports of drug abuse amongst the eligible participants. The abuse potential of S-ketamine is related to parenteral use and this part of the study will be conducted at the hospital under strict control. Furthermore, a well-known or suspected substance abuse potential such as excess alcohol consumption will lead to exclusion from the trial. Patients will continue on their usual analgaesic medication when the trial ends. This also applies to dropouts.

\section{Sample size}

The study is powered to detect a minimal difference of $30 \%$ between groups on the average pain diary score during 4 weeks of study treatment (primary end point). On the basis of an SD of $25 \%$ of the mean we determined that a study with 15 patients per group is needed to provide a power of $90 \%$, with the use of a two-sided significance level of 0.05 . Hence, the sample size is set at 20 patients per group to allow for possible dropouts.

\section{Data analysis}

The principal analysis of end points will be by intention-to-treat, meaning that all randomised patients are included in their initially assigned study arm, regardless of adherence to study protocol. Experimental end points will be by per-protocol, meaning that only patients completing the experimental setup are included. The primary end point will be compared between the treatment groups by mixed models, and subsequent analyses directed at the secondary, experimental and safety end points will be analysed using appropriate statistics.

\section{Data handling and record keeping}

The study is approved by the Danish Data Protection Agency. For each participants, a case report form will be kept in which data for the participants will be entered. Data will be stored under lock and key at Department of Gastroenterology and Hepatology, Aalborg University Hospital, for 5 years, under the responsibility of the principal investigator, Professor Asbjørn Mohr Drewes. All forms will be filled out during (or immediately after) the assessment of a participant and must be legible. Errors will be crossed out, corrections added and, next to the changes, date and initials will be entered. A patient identification list containing patient number, full name, social security number, study medication and treatment codes for all persons included in the study will be created. The list will be populated and updated by a project nurse or other competent person and stored at the same facility. The principal investigator will be required to maintain complete and accurate records to ensure that the execution of the study gets fully documented and the study data can be subsequently verified.

\section{Author affiliations}

${ }^{1}$ Mech-Sense, Department of Gastroenterology and Hepatology, Aalborg University Hospital, Aalborg, Denmark

${ }^{2}$ Department of Drug Design and Pharmacology, University of Copenhagen, Copenhagen, Denmark

${ }^{3}$ Department of Anesthesiology, Leiden University Medical Center, Leiden, The Netherlands

${ }^{4}$ Department of Anesthesiology, Pain and Palliative Medicine, Radboud University, Nijmegen Medical Centre, Nijmegen, The Netherlands

${ }^{5}$ Department of Health Science and Technology, Center for Sensory-Motor Interactions, Aalborg University, Aalborg, Denmark

${ }^{6}$ Mech-Sense, Department of Radiology, Aalborg University Hospital, Aalborg, Denmark

${ }^{7}$ Department of Clinical Medicine, Aalborg University, Aalborg, Denmark

Contributors JJ, SSO, AEO, AD, OW-S, JLP, AM, JBF and AMD conceived and designed the study and participated in logistical planning of the study. $\mathrm{JJ}$ drafted the initial version of the manuscript and made the initial data acquisition. AEO is continuing the acquisition of the data. SSO provided the statistical support for the sample size estimates and the design of the statistical analysis. All authors made significant contributions to the development and conceptualisation of the protocol. All authors reviewed the draft versions of this paper and have read and approved the final manuscript.

Funding The study is conducted as an investigator-initiated study with financial support from the Danish Council for Strategic Research.

\section{Competing interests None.}

Ethics approval The protocol is approved by the North Denmark Region Committee on Health Research Ethics (Reference-number: N-20130040) and the Danish Health and Medicines Authorities (EudraCT-number: 2013-003357-17).

Provenance and peer review Not commissioned; externally peer reviewed.

Open Access This is an Open Access article distributed in accordance with the Creative Commons Attribution Non Commercial (CC BY-NC 4.0) license, which permits others to distribute, remix, adapt, build upon this work noncommercially, and license their derivative works on different terms, provided the original work is properly cited and the use is non-commercial. See: http:// creativecommons.org/licenses/by-nc/4.0/

\section{REFERENCES}

1. Andersen BN, Pedersen NT, Scheel J, et al. Incidence of alcoholic chronic pancreatitis in Copenhagen. Scand $J$ Gastroenterol 1982;17:247-52.

2. Steer ML, Waxman I, Freedman S. Chronic pancreatitis. N Engl J Med 1995;332:1482-90.

3. Andren-Sandberg A, Ansorge C, Eiriksson K, et al. Treatment of pancreatic pseudocysts. Scand J Surg 2005;94:165-75.

4. Lieb JG II, Forsmark CE. Review article: pain and chronic pancreatitis. Aliment Pharmacol Ther 2009;29:706-19.

5. Olesen AE, Brokjaer A, Fisher IW, et al. Pharmacological challenges in chronic pancreatitis. World J Gastroenterol [Internet]. 2013;19:7302-7.

6. van Esch AA, Wilder-Smith $\mathrm{OH}$, Jansen JB, et al. Pharmacological management of pain in chronic pancreatitis. Dig Liver Dis 2006;38:518-26. 
7. Pasricha PJ. Unraveling the mystery of pain in chronic pancreatitis. Nat Rev Gastroenterol Hepatol 2012;9:140-51.

8. Whitcomb DC, Schneider A. Hereditary pancreatitis: a model for inflammatory disease of the pancreas. Best Pract Res Clin Gastroenterol 2002;16:347-63.

9. Ceyhan GO, Demir IE, Rauch U, et al. Pancreatic neuropathy results in "neural remodeling" and altered pancreatic innervation in chronic pancreatitis and pancreatic cancer. Am J Gastroenterol 2009;104:2555-65.

10. Paisley $\mathrm{P}$, Kinsella J. Pharmacological management of pain in chronic pancreatitis. Scot Med J 2014:59:71-9.

11. Coppel DL, Bovill JG, Dundee JW. The taming of ketamine. Anaesthesia 1973;28:293-6.

12. Noppers I, Niesters M, Aarts L, et al. Ketamine for the treatment of chronic non-cancer pain. Expert Opin Pharmacother 2010;11:2417-29.

13. Woolf CJ. Central sensitization: implications for the diagnosis and treatment of pain. Pain 2011;152(3 Suppl):S2-15.

14. Woolf CJ, Salter MW. Neuronal plasticity: increasing the gain in pain. Science 2000;288:1765-9.

15. Woolf CJ, American College of Physicians, American Physiological Society. Pain: moving from symptom control toward mechanism-specific pharmacologic management. Ann Intern Med 2004;140:441-51.
16. Bouwense SA, Buscher HC, van Goor H, et al. S-ketamine modulates hyperalgesia in patients with chronic pancreatitis pain. Reg Anesth Pain Med 2011;36:303-7.

17. Aaronson NK, Ahmedzai S, Bergman B, et al. The European Organization for Research and Treatment of Cancer QLQ-C30: a quality-of-life instrument for use in international clinical trials in oncology. J Natl Cancer Inst 1993;85:365-76.

18. Mendoza T, Mayne T, Rublee D, et al. Reliability and validity of a modified brief pain inventory short form in patients with osteoarthritis. Eur J Pain 2006;10:353-61.

19. Bloechle C, Izbicki JR, Knoefel WT, et al. Quality of life in chronic pancreatitis-results after duodenum-preserving resection of the head of the pancreas. Pancreas 1995;11:77-85.

20. Farrar JT, Young JP Jr, LaMoreaux L, et al. Clinical importance of changes in chronic pain intensity measured on an 11-point numerical pain rating scale. Pain 2001;94:149-58.

21. Beck AT, Ward $\mathrm{CH}$, Mendelson $\mathrm{M}$, et al. An inventory for measuring depression. Arch Gen Psychiatry 1961;4:561-71.

22. Vignaroli E, Pace EA, Willey J, et al. The Edmonton Symptom Assessment System as a screening tool for depression and anxiety. J Palliat Med 2006:9:296-303.

23. Rasmussen KG. Psychiatric side effects of ketamine in hospitalized medical patients administered subanesthetic doses for pain control. Acta Neuropsychiatr 2014;26:230-3. 